\title{
Die Validierung der Dimensional Obsessive-Compulsive Scale (DOCS) an einer deutschsprachigen Stichprobe
}

\author{
Jakob Fink-Lamotte ${ }^{a}$ Ina Jahn ${ }^{b} \quad$ Christian Stierle $^{c, d}$ Franziska Kühne ${ }^{e}$ \\ Tania Lincoln ${ }^{f}$ Katarina Stengler ${ }^{b}$ Cornelia Exner ${ }^{a}$ \\ ${ }^{a}$ Klinische Psychologie und Psychotherapie, Universität Leipzig, Leipzig, Deutschland; b Helios Park-Klinikum \\ Leipzig, Akademisches Lehrkrankenhaus der Universität Leipzig, Leipzig, Deutschland; ' IUBH Internationale \\ Hochschule, Bad Reichenhall, Deutschland; ${ }^{\mathrm{d} B l o m e n b u r g}$ Privatklinik, Selent, Deutschland; ${ }^{\mathrm{E} K l i n i s c h e ~ P s y c h o l o g i e}$ \\ und Psychotherapie, Universität Potsdam, Potsdam, Deutschland; ${ }^{f}$ Klinische Psychologie und Psychotherapie, \\ Universität Hamburg, Hamburg, Deutschland
}

\section{Schlüsselwörter}

Zwangsstörung · Dimensional Obsessive-Compulsive Scale (DOCS) · Selbstbeurteilungsfragebogen · Validierungsstudie

\section{Zusammenfassung}

Hintergrund: PatientInnen mit Zwangsstörungen werden in klinischer Forschung und Praxis häufig nicht erkannt oder fehldiagnostiziert, wodurch es zu verzögerten und unzureichenden Behandlungen kommt. Ein Aspekt, der maßgeblich dazu beiträgt, sind unter anderem unzureichende Screening- und Diagnostikinstrumente, da bestehende Fragebögen zur Erfassung von Zwangsstörungen zu einem großen Teil Limitationen hinsichtlich ihrer psychometrischen Qualitäten und ihrer Aktualität unterliegen. Mit der Dimensional Obsessive-Compulsive Scale (DOCS) steht im englischsprachigen Raum seit mehreren Jahren bereits ein vielversprechendes Instrument zur Erfassung von Zwangsstörungen zur Verfügung. Patienten und Methoden: Ziel der vorliegenden Arbeit war es, erstmalig die Faktorstruktur sowie die psychometrischen Eigenschaften der deutschsprachigen DOCS-Version zu überprüfen. Hierzu wurden 177 PatientInnen (107 mit Zwangsstörung, 30 mit Angststörungen und 40 mit Depression) sowie eine nicht-klinische Kontrollgruppe mit 223 Probanden untersucht. Ergebnisse: Die vierfaktori-

Jakob Fink-Lamotte und Ina Jahn teilen sich die Erstautorenschaft.

karger@karger.com www.karger.com/ver

Karger $\stackrel{2}{*}$

BOPEN ACCESS
(C) 2020 The Author(s)

Published by S. Karger AG, Basel

This is an Open Access article licensed under the Creative Commons Attribution-NonCommercial-4.0 International License (CC BY-NC) (http://www.karger.com/Services/OpenAccessLicense), applicable to the online version of the article only. Usage and distribution for commercial purposes requires written permission. elle Originalstruktur der DOCS konnte in explorativen und konfirmatorischen Faktoranalysen repliziert werden. Interne Konsistenzen und die zeitliche Stabilität der DOCS fielen akzeptabel bis sehr gut aus. Die Konstruktvalidität der DOCSGesamtskala fiel zufriedenstellend bis gut aus. Die Kriteriumsvaliditäten fielen bedingt zufriedenstellend aus. Die diagnostische Genauigkeit der DOCS fiel befriedigend aus. Schlussfolgerung: Die deutsche Version der DOCS ist ein robustes, reliables und erstmals validiertes Instrument zur dimensionalen Beschreibung des Schweregrads von Zwangsstörungen.

(c) 2020 The Author(s) Published by S. Karger AG, Basel

\section{The Validation of the Dimensional Obsessive- Compulsive Scale (DOCS) on a German-Speaking Sample}

\section{Keywords}

Obsessive-compulsive disorder - Dimensional Obsessive-Compulsive Scale (DOCS) · Self-report questionnaire Validation

\section{Abstract}

Background: Patients with obsessive-compulsive disorder are often misdiagnosed in clinical research and practice, resulting in delayed and inadequate treatment for 
patients. Deficient screening and diagnostic instruments contribute to this, since existing questionnaires for obsessive-compulsive disorders are subject to limitations with regard to their psychometric qualities and their topicality. The Dimensional Obsessive-Compulsive Scale (DOCS) is a promising instrument for assessing obsessive-compulsive disorders. Patients and Methods: The aim of the present study was to examine for the first time the factor structure and the psychometric properties of the German-language DOCS version. A total of 177 patients (107 with obsessive-compulsive disorder, 30 with anxiety disorder, and 40 with depression) and a non-clinical control group with 223 subjects were examined. Results: The original four-factor structure of the DOCS could be replicated in explorative and confirmatory factor analyses. Internal consistency and temporal stability of the DOCS were acceptable to very good. The construct validity of the overall DOCS scale was satisfactory to good. The criterion validities were conditionally satisfactory. The diagnostic accuracy of the DOCS was satisfactory. Conclusion: The German version of the DOCS is a robust, reliable, and for the first time validated instrument for the assessment of obsessive-compulsive disorders.

(c) 2020 The Author(s) Published by S. Karger AG, Base

\section{Einleitung}

Zwangsstörungen sind mit einer Lebenszeitprävalenz von 1-3\% [Kessler et al., 2005; Ruscio et al., 2010] relativ häufige psychische Störungen. Die Störung bleibt oft unerkannt oder wird häufig fehldiagnostiziert [Heyman et al., 2001], was auch durch hohe Komorbiditäten mit anderen Angststörungen und Depressionen [Ruscio et al., 2010] erklärt wird. Die Herausforderungen in der Diagnostik von Zwangsstörungen liegen auch im heterogenen Symptombild [Abramowitz et al., 2010]. Es zeigen sich dabei verschiedene Symptomcluster, die beispielsweise Zwangssymptome wie Waschen und Säubern, Kontrollieren, Ordnen und inakzeptable Gedanken umfassen [Mataix-Cols et al., 2005]. Die Symptomcluster können, müssen aber nicht, Zwangsgedanken und Zwangshandlungen umfassen. So können kontaminationsbezogene Zwangsgedanken über Zwangshandlungen wie Waschen, Säubern und/oder Desinfizieren neutralisiert werden. Bei Zwangsgedanken bezüglich einer subjektiv erhöhten Verantwortung für Fehler aller Art werden dagegen häufig Kontrollhandlungen zur Neutralisierung verwendet. Zwangsgedanken bezüglich Symmetrie und Ordnung können mit Zwangshandlungen wie Anordnen und Ordnen neutralisiert werden. Und Zwangsgedanken über Sexualität, Religion und Gewalt werden oft über gedankliche Rituale neutralisiert.
Diese Heterogenität der Zwangsstörung führt in der Diagnostik vor allem bei Selbstberichtsfragebögen $\mathrm{zu}$ Problemen. Das Fremdbeurteilungsinstrument YaleBrown Obsessive-Compulsive Scale (Y-BOCS) [Hand und Büttner-Westphal, 1991] gilt bislang meist als Goldstandard zur Diagnostik der Zwangsstörung [Storch et al., 2010]. Die Y-BOCS zeigt in der Originalversion von Goodman et al. [1989a] eine sehr hohe Interrater-Reliabilität. Daneben zeigt sich eine gute konvergente Validität und eine moderate diskriminante Validität [Goodman et al., 1989b]. Bei der Bewertung der Symptomatik mit der Y-BOCS nimmt der Interviewer eine Zusammenführung der dimensionalen (Heterogenität im Erscheinungsbild) und der quantitativen Aspekte der Zwangssymptomatik (Schweregrad) vor. Dies erfolgt über die Erfassung aller Symptome mittels einer Checkliste und der darauffolgenden Einschätzung von Zeitdauer, Vermeidung, Leid, Beeinträchtigung, und Widerstand. Hierbei geht allerdings der qualitative Aspekt der kategorialen Diagnostik verloren, weswegen die genaue Ausprägung jedes Symptoms nicht dargestellt werden kann.

Zur Erfassung von Zwangssymptomen gibt es bereits eine Reihe von bestehenden Selbstbeurteilungsfragebögen, wie das Obsessive-Compulsive Inventory (OCI) [Foa et al., 2002] und das Padua Inventory (PI) [Sanavio, 1988; Gönner et al., 2007, 2010]. Zusätzlich ist im deutschsprachigen Raum das Hamburger Zwangsinventar (HZI) [Zaworka et al., 1983; Klepsch et al., 1991] verbreitet. Die Fragebögen sind ökonomisch in der Durchführung und haben gute psychometrische Qualitäten, zeigen allerdings eine Reihe von Limitationen, die nachfolgend aufgelistet sind:

- Die Zusammenführung der dimensionalen (Heterogenität im Erscheinungsbild) und der quantitativen Aspekte der Zwangssymptomatik (Schweregrad) fehlt. Zum Beispiel erfassen OCI-R und PI die Störungsschwere nur eindimensional und additiv über verschiedene Arten von Zwangssymptomen (Beeinträchtigung durch das Symptom). Das führt zu einer Überschätzung der Schwere, wenn PatientInnen viele verschiedene Symptome haben.

- Zwangsgedanken und Zwangshandlungen werden getrennt beurteilt, obwohl sie häufig einen funktionalen Bezug zueinander aufweisen. So werden kontaminationsbezogene Zwangsgedanken und Zwangshandlungen wie Waschen, Säubern und/oder Desinfizieren in den meisten Fragebögen über zwei oder mehrere Fragen beurteilt. In der Funktionalität der Störung dienen Zwangshandlungen häufig dazu, Zwangsgedanken zu neutralisieren. Aus diesem Grund sollten diese also in der Schweregradbeurteilung gemeinsam betrachtet werden.

- Vermeidungsstrategien werden gar nicht oder sehr spezifisch erfasst. 
- Oft erfolgt nur die Abfrage einiger weitverbreiteter Symptome, was den Schweregrad aber unterschätzen könnte, wenn Patienten durch einzelne Symptome sehr stark eingeschränkt sind.

- Es zeigt sich eine nicht zufriedenstellende, geringe diskriminante Validität der Y-BOCS bezogen auf die depressive Symptomatik [Jacobsen et al., 2003].

- Generell weisen die Instrumente eine niedrige diskriminante Validität zu Maßen der Depression auf [Woody et al., 1995; Foa et al., 2002].

- Die Reliabilität der Y-BOCS als Fremdratingversion ist zudem abhängig vom Trainingsstand der Interviewer und relativ zeitaufwendig.

- Die Symptomchecklisten (HZI und HZI-K) sind zusätzlich durch dichotome Antwortformate beschränkt.

- Daneben entspricht beispielsweise der OCI-R nicht der neuesten klassifikatorischen Differenzierung von Zwangsstörungen und Zwangsspektrumsstörungen. So wird Horten mit in den Selbstbeurteilungsfragebogen eingeschlossen, was nicht mehr der aktuellen Klassifikation des DSM-5 [American Psychiatric Association, 2013] entspricht, wo Horten als eigenes Störungsbild den Zwangsspektrumsstörungen zugeordnet wird.

Um diese Limitationen zu überwinden, haben Abramowitz und seine Kollegen 2010 die Dimensional Obessive-Compulsive Scale (DOCS) entwickelt. Es handelt sich dabei um ein Selbstbeurteilungsinstrument mit 20 Items zu den vier oben bereits benannten Subtypen der Zwangsstörung, die als Kategorien abgefragt werden: Kontamination, Verantwortung für Unheil, Inakzeptable Gedanken und Symmetrie. Im Sinne der neuen klassifikatorischen Einordnung im DSM-5 [American Psychiatric Association, 2013] wird das Horten hierbei nicht eingeschlossen. Um die Kategorien deutlich zu machen, werden zu Beginn jeder Kategorie Beispiele genannt, die in diese Kategorie fallen, z.B. in der Kategorie "Kontamination" das Beispiel: "Das Gefühl, dass Sie verunreinigt sind, weil Sie sich an einem bestimmten Ort aufgehalten haben (wie z.B. der Toilette)." Hiernach werden zu jeder Symptomkategorie Fragen zu Zeitbedarf, Vermeidung, Leid, Beeinträchtigung und Widerstand auf einer 5-stufigen Skala erfragt, die ähnlich auch die Basis für die quantitative Erfassung innerhalb der Y-BOCS bilden. Somit wird die Symptomschwere mehrdimensional erfasst.

Die DOCS zeigt im Original eine gute psychometrische Qualität und ist sowohl für den klinischen, wie auch für den nicht-klinischen Bereich anwendbar [Abramowitz et al., 2010]. Die DOCS ist bereits in verschiedene Sprachen übersetzt und validiert worden, beispielsweise Chinesisch [Wang et al., 2012], Spanisch [López-Solà et al., 2014], Isländisch [Ólafsson et al., 2013] und Italienisch [Melli et al., 2015]. Im Großen und Ganzen bestä- tigen die Übersetzungen die guten psychometrischen Eigenschaften der Originalversion. Nach unserem Kenntnisstand liegt bisher keine deutsche Übersetzung der Langversion vor. Das Vorliegen einer reliablen und validen deutschen Version wäre sowohl zur Diagnostik im klinisch-praktischen Bereich wie auch für die Forschung eine wichtige Ergänzung, insbesondere für interkulturelle Vergleiche. Wie in der Originalstudie [Abramowitz et al., 2010] wird in der vorliegenden Studie die deutsche Langversion auf diagnostische Genauigkeit empirisch geprüft. Erstens werden ähnlich gute psychometrische Eigenschaften der deutschen Version wie in der Originalstudie vorhergesagt. Zweitens kann erwartet werden, dass die DOCS eine ähnlich gute Validität und diagnostische Genauigkeit aufweist wie PI-PR und OCI-R (mit Horten).

\section{Methoden}

DOCS - Itementwicklung, -beschreibung und -übersetzung

Initial entwickelt wurden die DOCS-Items von Jonathan S. Abramowitz und Brett J. Deacon [Abramowitz et al., 2010] mit dem Ziel, die am häufigsten gefundenen Symptomcluster der Zwangsstörung [McKay et al., 2004; Mataix-Cols et al., 2005] abzudecken. Daraus entstanden 20 Items, die sich auf vier Symptomcluster (Kontamination, Verantwortung, Symmetrie und Ordnung und Inakzeptable Gedanken) beziehen. Innerhalb dieser Symptomcluster wird über jeweils fünf Items (a) die Dauer, (b) das Vermeidungsverhalten, (c) das Leiden, (d) die Beeinträchtigung und (e) der persönliche Widerstand auf einer 5-stufigen Skala mit variierenden Schweregradbeschreibungen $(0=$ keine Beschwerden/Beeinträchtigungen, 4 = extreme Beschwerden/Beeinträchtigungen) erfasst. Die einzelnen Items sind, wie in der Originalversion, auch im Deutschen inhaltlich auf die Symptomcluster angepasst. Die Befragung bezieht sich auf den letzten Monat. Die deutschsprachige Version der DOCS ist online unter www.karger.com/ doi/10.1159/000510093 verfügbar.

Die DOCS wurde im Rahmen einer an der Universität Leipzig durchgeführten Vorstudie von zwei deutschen Muttersprachlerinnen mit sehr guten Englischkenntnissen aus dem englischen Original ins Deutsche übersetzt. Aus den beiden entstandenen Versionen wurde, nach Prüfung auf Übereinstimmung, eine deutsche Version erstellt. Nach dem von Brislin [1970] empfohlenen Vorgehen der back translation technique wurde die deutsche Version von einer bilingualen Muttersprachlerin ins Englische rückübersetzt. Die so entstandene Version wurde von Abramowitz auf Äquivalenz mit der Originalversion überprüft. Nachdem kleine Abweichungen angepasst worden waren, gab Abramowitz sein Einverständnis für die semantische Übereinstimmung der deutschen Version der DOCS mit der englischen Originalversion.

\section{Stichprobenbeschreibung}

Die Diagnosen der PatientInnen wurden in allen Einrichtungen von approbierten PsychotherapeutInnen mithilfe des Strukturierten Klinischen Interviews für DSM-IV (SKID) [Wittchen et al., 1997] gestellt und in ICD-10-Diagnosen überführt. Die PsychotherapeutInnen haben die Ein- und Ausschlusskriterien geprüft, weswegen nur PatientInnen an der Studie teilnahmen, die von den PsychotherapeutInnen anhand dieser Kriterien als geeignet eingeschätzt wurden. Es hätten auch solche PatientInnen, de- 
Tabelle 1. Demografische Merkmale der TeilnehmerInnen je Gruppen

\begin{tabular}{|c|c|c|c|c|}
\hline & \multicolumn{4}{|l|}{ Gruppe } \\
\hline & Zwangsstörung & Angststörung & Depression & Kontrollgruppe \\
\hline$n$ & 107 & 30 & 40 & 223 \\
\hline Alter (SD); Range, Jahre & $36,0(11,63) ; 18-62$ & $32,7(8,21) ; 21-54$ & $47,45(13,81) ; 23-78$ & $28,0(10,6) ; 18-78$ \\
\hline Geschlecht, w:m (\%w) & $69: 38(64 \%)$ & $16: 14(53 \%)$ & $23: 17(58 \%)$ & $175: 48(78,5 \%)$ \\
\hline \multirow[t]{5}{*}{ Diagnosen ICD-10 } & F42.1: $15(14 \%)$ & F40.01: $14(47 \%)$ & F32.0: $5(13 \%)$ & - \\
\hline & F42.2: $75(72 \%)$ & F40.1: $10(33 \%)$ & F32.1: $7(18 \%)$ & - \\
\hline & & F41.0: $6(20 \%)$ & F32.2: $1(3 \%)$ & - \\
\hline & & & F33.1: $15(38 \%)$ & - \\
\hline & & & F33.2: $13(33 \%)$ & - \\
\hline Komorbiditäten & $63(59 \%)$ & $14(47 \%)$ & $31(78 \%)$ & - \\
\hline \multicolumn{5}{|l|}{ Erhebungsort } \\
\hline PKL & $62(58 \%)$ & - & - & - \\
\hline SK & $45(42 \%)$ & $3(10 \%)$ & $40(100 \%)$ & - \\
\hline HSA & - & $27(90 \%)$ & - & - \\
\hline Pharmakologische Behandlung & $83(78,2 \%)$ & $20(67 \%)$ & $29(73 \%)$ & - \\
\hline
\end{tabular}

Bei den PatientInnen mit einer Zwangsstörung wurden PatientInnen mit Haupt- oder Nebendiagnose Zwangsstörung eingeschlossen. PKL, Zwangsambulanz des Park-Klinikums Leipzig; SK, Schön-Klinik Bad-Bramstedt; HSA, Psychotherapeutische Hochschulambulanz der Universität Leipzig.

ren Erstdiagnose eine andere psychische Erkrankung darstellte, eingeschlossen werden können, dies war aber nicht der Fall. Alle PatientInnen mit einer Zwangssymptomatik hatten eine Erstdiagnose Zwangsstörung. Aufgrund der Ausschlusskriterien Schwangerschaft, derzeitiger Tavor-Entzug, hirnorganische Störung und/ oder Wahnvorstellungen und Halluzinationen wurden drei PatientInnen von der Studienteilnahme ausgeschlossen (einmal Tavor-Entzug, zweimal wegen einer falschen Zieldiagnose).

Insgesamt nahmen 400 Personen an der vorliegenden Validierungsstudie teil. Darunter waren 223 nicht-klinische TeilnehmerInnen, 107 PatientInnen mit Zwangsstörung, 30 PatientInnen mit Angststörungen und 40 PatientInnen mit Depression. Die stationäre und ambulante Behandlung basierte auf den Prinzipien der KVT und beinhaltete Exposition mit Reaktionsverhinderung. Die Daten der nicht-klinischen Kontrollgruppe $(n=223)$ wurden über soziale Netzwerke und E-Mail-Verteiler erhoben. Die demografischen Merkmale der TeilnehmerInnen, aufgeschlüsselt nach den einzelnen Gruppen, und die Erhebungsorte sind in Tabelle 1 dargestellt. Eine Sub-Stichprobe von 107 TeilnehmerInnen nahm drei bis zwölf Wochen nach dem erstmaligen Test an einer ReTestung teil. Es liegt ein positives Votum der Ethikkommission der Medizinischen Fakultät der Universität Leipzig vom 23.06.2016 vor (217/16-ek).

\section{Messinstrumente}

Zusätzlich zur DOCS (die oben genauer beschrieben wurde) wurden die nachfolgenden Fragebögen in der Studie erhoben. Zur Erfassung der konvergenten Validität wurden die Yale-Brown Obsessive-Compulsive Scale (Y-BOCS), das Obsessive-Compulsive Inventory-Revised (OCI-R) und das Padua Inventory-Palantine Revision (PI-PR) verwendet. Zur Erfassung der diskriminanten Validität wurden das Beck-Depressions-Inventar (BDI-II), das Beck-Angst-Inventar (BAI) und die Social Interaction Anxiety Scale (SIAS) verwendet.

\section{Demographische Daten}

Erfragt wurden Geschlecht, Alter, höchster Schul- und Berufsabschluss, derzeitig ausgeübter Beruf, aktuelle Wohnsituation,
Anzahl der Sozialkontakte und die aktuelle Medikation sowie die Ausschlusskriterien Hirnorganische Erkrankungen, Posttraumatische Belastungsstörung, Wahnvorstellungen oder Halluzinationen, Tavor-Entzug und Schwangerschaft.

\section{Die Yale-Brown Obsessive-Compulsive Scale (Y-BOCS)}

In der vorliegenden Studie wurde die Y-BOCS in der deutschen Selbstratingversion des von Goodman et al. [1989a] entwickelten und von Hand und Büttner-Westphal [1991] ins Deutsche übersetzten Fremdbeurteilungsinstruments zur Erfassung des Schweregrades von Zwangsstörungen verwendet. Die Y-BOCS besteht aus 10 Items und erfasst Zwangshandlungen und Zwangsgedanken getrennt voneinander, wobei die Schwere der Symptomatik über fünf Bereiche (Zeit, Vermeidung, Leid, Widerstand, Beeinträchtigung) in einem fünfstufigen Antwortformat erfragt wird (0 $=$ keine Symptome bis $4=$ schwere Symptome). Die deutsche Version der Y-BOCS verfügt über sehr gute psychometrische Eigenschaften [Jacobsen et al., 2003]. In der vorliegenden Studie wurde die Y-BOCS als Selbstratingverfahren eingesetzt [Schaible et al., 2001].

Obsessive-Compulsive Inventory-Revised (OCI-R)

Das OCI-R wurde in der vorliegenden Studie in der deutschen Kurzversion [Gönner et al., 2007] des ursprünglich von Foa et al. [2002] entwickelten Selbstbeurteilungsinstruments zur multidimensionalen Erfassung von Zwangssymptomen verwendet. Das OCI-R erfasst die sechs Symptomdimensionen Waschen, Kontrollieren, Ordnen, Zwangsgedanken, Horten und Mentales Neutralisieren. Jede Dimension wird mithilfe von drei Items erhoben, sodass das OCI-R insgesamt 18 Items umfasst, die auf einer fünfstufigen Antwortskala eingeschätzt werden ( $0=$ gar nicht bis $4=$ sehr stark). Die guten psychometrischen Eigenschaften des OCI-R konnten in der deutschen Version weitestgehend repliziert werden. Einzig die geringe diskriminante Validität bezüglich depressiver Symptome ist nicht zufriedenstellend. Sie fällt in der deutschen Übersetzung aber besser aus als in der Originalversion [Gönner et al., 2007]. Es stehen Cut-Off-Werte für die Gesamtskala sowie die Subskalen zur Verfügung. 
Padua Inventory-Palatine Revision (PI-PR)

Das PI-PR [Gönner et al., 2010] ist die revidierte, deutsche Kurzversion des PI [Sanavio, 1988] mit 24 Items zur Erfassung von sechs Symptomdimensionen von Zwang: Kontamination und Waschen (4 Items), Kontrollieren (6 Items), Zahlen (3 Items), Ankleiden und Körperpflege (3 Items), Rumination (3 Items) sowie Bedrohliche Zwangsgedanken und Impulse (5 Items). Die Aussagen zum Schweregrad der Symptome werden auf einer fünfstufigen Likert-Skala eingeschätzt $(0=$ gar nicht bis $4=$ sehr stark $)$. Das PI-PR hat zufriedenstellende Reliabilitäts- und Validitätswerte, einzig die konvergente Validität mit dem Y-BOCS-Gesamtwert fällt moderat aus.

\section{Beck-Depressions-Inventar (BDI-II)}

Das BDI-II ist die deutsche, revidierte Version [Hautzinger et al., 2006] des von Beck et al. [1961] entwickelten Selbstbeurteilungsinstruments zur Erfassung des Schweregrads der depressiven Symptomatik. Das BDI-II umfasst 21 Items. Die Antwortmöglichkeiten sind nach aufsteigendem Schweregrad des Symptoms auf einer vierstufigen Skala $(0=$ keine Symptomatik bis $3=$ schwere Symptomatik) geordnet. Das BDI-II zeigt insgesamt gute psychometrische Gütekriterien [Kühner et al., 2007].

Beck-Angst-Inventar (BAI)

Das BAI ist die deutsche Version [Prinz und Petermann, 2009] des von Beck et al. [1988] entwickelten Selbstbeurteilungsinstruments zur Erfassung der Schwere von Angstsymptomen. Das BAI besteht aus 21 vierstufigen $(0=$ überhaupt nicht bis $3=$ stark/ich konnte es kaum aushalten) deskriptiven Items. Es liegen sehr gute psychometrische Kriterien der Originalversion vor [Beck et al., 1988]. Die deutsche Version weist eine gute Reliabilität und zufriedenstellende Validitäten auf [Prinz und Petermann, 2009].

\section{Social Interaction Anxiety Scale (SIAS)}

Das Selbstbeurteilungsinstrument SIAS wurde von Mattick und Clarke [1998] zur Erfassung allgemeiner Ängste vor sozialen Interaktionen entwickelt und wurde in der vorliegenden Studie in der deutschen Version von Stangier et al. [1999] verwendet. Die SIAS besteht aus 20 Aussagen zu sozialen Situationen, welche auf einer fünfstufigen Ratingskala $(0=$ überhaupt nicht zutreffend bis $4=$ sehr stark zutreffend) eingeschätzt werden. Die SIAS ist ein reliables und valides Instrument zur Erfassung kognitiver, affektiver und behavioraler Komponenten von sozialer Angst [Mattick und Clarke, 1998] und weist auch in der deutschen Übersetzung sehr gute psychometrische Qualitäten auf, die mit denen der Originalversion vergleichbar sind [Stangier et al., 1999].

\section{Ablauf der Untersuchung}

Klinische Stichprobe

Die Erhebung für die Validierung wurde an der Schön-Klinik Bad Bramstedt, am Helios Parkkrankenhaus Leipzig, der Arbeitsgruppe Klinische Psychologie und Psychotherapie Universität Leipzig, der Psychotherapeutischen Hochschulambulanz der Universität Leipzig sowie dem Leipziger Ausbildungsinstitut für Psychologische Psychotherapie durchgeführt. Die TeilnehmerInnen füllten die oben beschriebenen Fragebögen im PaperPencil-Verfahren aus, nachdem ein Überblick über das Vorhaben, die Ziele und den Ablauf der Studie gegeben worden war und sie ihr Einverständnis zur Teilnahme gegeben hatten. Die PatientInnen wurden gebeten, sich für die Beantwortung der Fragebögen Zeit und Ruhe zu nehmen. Sie füllten die Fragebögen zu unterschiedlichen Zeitpunkten ihrer derzeitigen Behandlung aus.
Nicht-klinische Stichprobe

Der Gesamtfragebogen wurde von der nicht-klinischen Stichprobe online am Computer ausgefüllt. Ihnen wurde ein Überblick über das Vorhaben, die Ziele und den Ablauf der Studie gegeben. Nachdem die TeilnehmerInnen ihr Einverständnis zur Teilnahme an der Studie erklärt hatten, wurden ihnen alle Selbstbeurteilungsfragebögen mit Ausnahme der Y-BOCS präsentiert. Die technische Umsetzung erfolgte mittels Unipark (Questback GmbH).

Test-Retest-Sample

Eine Sub-Stichprobe von 107 Teilnehmenden aus der nicht-klinischen Stichprobe nahm nach Einladung via E-Mail an einem Retest teil, für den von ihnen ausschließlich die DOCS beantwortet wurde.

\section{Datenaufbereitung und statistische Analyse}

Für jeden Teilnehmenden wurden die Summenwerte für jeden Fragebogen gebildet, wobei bei der DOCS, beim PI-PR und beim OCI-R zusätzlich zu den Gesamtwerten Summenwerte der Subskalen gebildet wurden. Da ein Teil der klinischen Stichprobe ( $n=$ $58 ; 32,77 \%$ ) das Item 10 des BAI (nervös) nicht beantwortet hatte, wurden diese Werte durch den jeweiligen Skalenmittelwert ersetzt. Andere fehlende Werte, die aufgrund des Paper-Pencil-Verfahrens ausschließlich in der klinischen Stichprobe vorkamen, wurden durch den Itemmittelwert desselben Items in der jeweiligen Stichprobe korrigiert, wenn nicht mehr als 30\% der Items pro Fragebogen fehlten. Bei insgesamt drei Studienteilnehmenden waren mehr als 30\% des jeweiligen Fragebogens unbeantwortet, sodass diese Werte nach Wirtz [2004] nicht ersetzt wurden. Diese Studienteilnehmenden wurden unter paarweisem Ausschluss in die Berechnungen miteinbezogen.

Danach wurde zuerst die faktorielle Struktur der DOCS untersucht. Die nicht-klinische Stichprobe wurde dazu zufällig in zwei Gruppen aufgeteilt. Die Faktorenstruktur der DOCS wurde wie im Originalartikel sowohl über eine explorative Faktorenanalyse (EFA) wie auch über eine konfirmatorische Faktorenanalyse (KFA) überprüft [Brown, 2006]. Hierfür wurden die R-Pakete psych (Version 1.9.12) [Revelle, 2019] und lavaan [Rosseel, 2012] verwendet. Mit der ersten Hälfte der Stichprobe wurde die untere Ordnung der Faktorenstruktur der DOCS mithilfe einer EFA erfasst. Danach wurde die Modellstruktur mithilfe der zweiten Hälfte der nicht-klinischen Stichprobe $(n=112)$ und mithilfe der zusammengenommenen klinischen Stichprobe (Zwangs-, Angst- und depressive PatientInnen, $n=177$ ) im Rahmen von zwei KFAs überprüft. Die klinischen Stichproben wurden wie in der Originalarbeit zusammengefasst, um die zugrundeliegende Stichprobengröße zu maximieren.

Hiernach wurden die Trennschärfe der Items und die interne Konsistenz mittels Cronbachs a [Cronbach, 1951] geprüft. Die Retest-Reliabilität im Sinne der zeitlichen Stabilität der DOCS wurde für die nicht-klinische Stichprobe berechnet. Drittens wurden die konvergente und diskriminante Konstruktvalidität und die Kriteriumsvalidität berechnet. Viertens wurde die diagnostische $\mathrm{Ge}$ nauigkeit mithilfe von Receiver Operating Characteristic (ROC) Analysen berechnet und fünftens die Wahl eines optimalen CutOff-Werts berechnet. Um hierbei die Stichprobengröße zu berücksichtigen, haben wir die Spezifitäts- und Sensitivitätsdaten mithilfe von lokaler Regression geglättet. Da mit Bezug auf die Studie von Abramowitz et al. [2010] die Balance von Spezifität und Sensitivität berücksichtigt werden sollte, wurde der Datenpunkt ausgewählt, bei dem Sensitivität und Spezifität die geringste Abweichung haben. Berechnet haben wir dies durch das Minimum der voneinander subtrahierten geglätteten Absolutwerte der Sensitivität und geglätteten Absolutwerte der Spezifität ( $\min (\mathrm{abs}(\mathrm{Sen})$ - abs(Spe))). Hierzu wurde dann der Cut-Off-Wert bestimmt. Die statistische Auswertung wurde mit dem Programm R [R Core Team, 2019] durchgeführt. 
Tabelle 2. Ladungen und Standardfehler (SE) für das finale Vierfaktorenmodell der deutschen DOCS im Rahmen der konfirmatorischen Faktorenanalyse mit der Kontrollgruppe und der klinischen Stichprobe

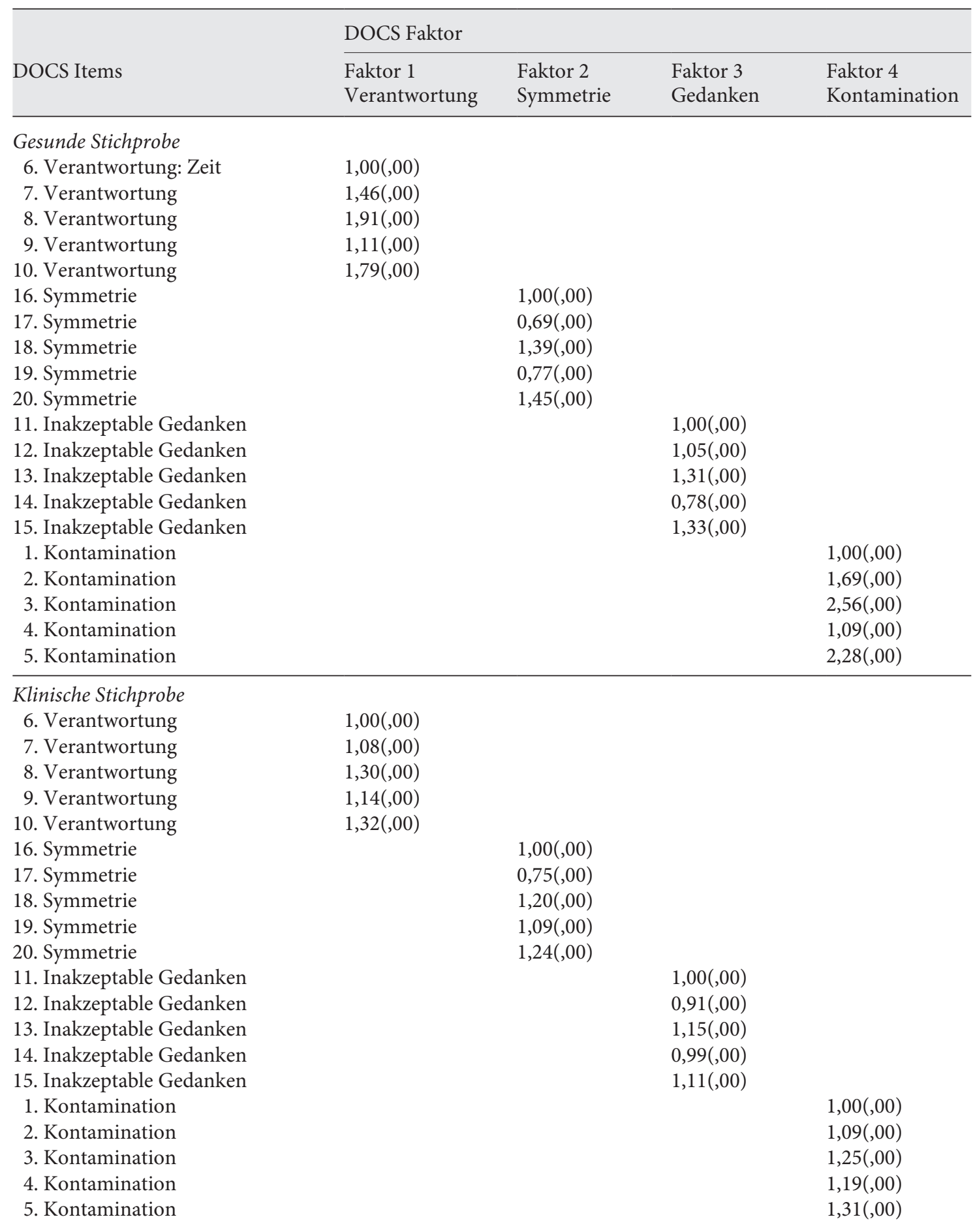

\section{Ergebnisse}

EFA anhand der Daten der nicht-klinischen

\section{Stichprobe}

Mit der Hälfte der Daten der nicht-klinischen Stichprobe wurde eine EFA mit obliquer Transformation (Promax) berechnet. Die fünf ersten Eigenwerte nach der
Rotation betrugen $6,61,2,25,1,54,1,07$ und 0,46 und können insgesamt 57\% der Varianz aufklären. Das KaiserMeyer-Olkin-Kriterium lag für alle Items bei $>0,75$; ebenso war der Bartletts-Test signifikant $\left(\chi^{2}(190)=1\right.$ '264,44; $p<0,001)$. Diese Ergebnisse unterstützen das Vorliegen einer Vierfaktorenstruktur der DOCS [Field, 2014]. Weiterhin laden alle Items nur auf einem Faktor. 
Tabelle 3. Cronbachs a und Pearsons Korrelationen (Subskalen mit Gesamtskala)

\begin{tabular}{lllll}
\hline \multicolumn{2}{l}{ GoCS-Skala } & \multicolumn{3}{l}{} \\
\cline { 2 - 5 } Zwangsstörung & Angststörung & Depression & Kontrollgruppe \\
\hline$N$ & 107 & 30 & 40 & 223 \\
\hline Cronbachs $\alpha$ & & & & \\
Gesamtskala & 0,93 & 0,93 & 0,91 & 0,91 \\
Verantwortung & 0,95 & 0,89 & 0,94 & 0,86 \\
Inakzeptable Gedanken & 0,95 & 0,93 & 0,95 & 0,88 \\
Symmetrie & 0,96 & 0,90 & 0,90 & 0,89 \\
Kontamination & 0,97 & 0,90 & 0,83 & 0,78 \\
\hline Korrelationen der Subskalen mit & dem DOCS-Gesamtwert & & \\
Verantwortung & $0,82^{*}$ & $0,76^{*}$ & $0,77^{*}$ & $0,77^{*}$ \\
Symmetrie & $0,71^{*}$ & $0,74^{*}$ & $0,75^{*}$ & $0,74^{*}$ \\
Inakzeptable Gedanken & $0,60^{*}$ & $0,91^{*}$ & $0,77^{*}$ & $0,77^{*}$ \\
Kontamination & $0,69^{*}$ & $0,64^{*}$ & $0,53^{*}$ & $0,73^{*}$ \\
\hline
\end{tabular}

Dargestellt sind Cronbachs a für den DOCS-Gesamtscore und für die DOCS-Subskalen für jede Stichprobengruppe sowie die Korrelationen der Subskalen mit dem DOCS-Gesamtscore. ${ }^{*} p<0,012$ (Bonferroni-korrigiertes Signifikanzniveau).

KFA anhand der Daten der zweiten Hälfte der nichtklinischen Stichprobe

Um die Faktorenstruktur zu verifizieren und dabei der Überschätzung des Modell-Fits in größeren Stichproben entgegenzuwirken [Bollen, 1989], wurden KFAs mit der zweiten Hälfte der nicht-klinischen Stichprobe durchgeführt. Es wurde eine Goodness-of-Fit-Analyse anhand verschiedener Indizes vorgenommen [s. Hu und Bentler, 1999; Hooper et al., 2008; Abramowitz et al., 2010]. Dabei wurden die folgenden Werte zugrunde gelegt: standardisiertes Root-Mean, Residual (akzeptabel bis gut: $0,06 \leq$ SRMR $\leq 0,08$ ), Root-Mean-Square Error-of-Approximation (akzeptabel bis gut 0,06 $\leq \mathrm{RM}$ SEA $\leq 0,08$ ), Comparative-Fit-Index (akzeptabel bis gut : $0,95 \geq$ CFI $\geq 0,9$ ) und Tucker-Lewis-Index (gut, TLI $\geq 0,95$ ). Die Goodness-of-Fit-Indizes der KFA sprechen für eine akzeptable bis gute Modellgüte für diese Gruppe $\left(\chi^{2}(164, n=112)=279,77 ; p<0,001 ; \chi^{2} / \mathrm{df}=1,89 ; \mathrm{RM}-\right.$ $\mathrm{SEA}=0,08$; $\mathrm{CFI}=0,91$; TLI $=0,89$ ). Die Goodness-ofFit-Indizes für ein Modell mit einem Faktor zweiter Ordnung sprechen für eine akzeptable bis gute Modellgüte $\left(\chi^{2}(164, n=112)=284,78, p<0,001 ; \chi^{2} / \mathrm{df}=1,73\right.$; RMSEA $=0,08$; CFI $=0,91$; TLI $=0,989)$. Alle Faktoren der ersten Ordnung luden stark auf dem Faktor der höheren Ordnung (Ladungen zwischen 1,00 und 2,46). Da die höhere Ordnung nicht zu einer signifikanten $\mathrm{Ab}$ nahme der Modellanpassung führte, kann zusammengefasst werden, dass das Modell die Korrelationen zwischen den Faktoren erster Ordnung gut wiedergibt [Brown, 2006].
KFA anhand der Daten der klinischen Stichprobe

Wie in der Originalstudie sollte die in der nicht-klinischen Stichprobe dokumentierte Struktur der DOCS auch in der klinischen Stichprobe vorliegen. Auch für diese KFA war die Modellgüte akzeptabel bis gut $\left(\chi^{2}(164\right.$, $n=177)=309,82 ; p<0,001 ; \chi^{2} / \mathrm{df}=1,67 ; \mathrm{RMSEA}=0,07$; $\mathrm{CFI}=0,97$; TLI $=0,96)$. Auch der Model-Fit der zweiten Ordnung ist als akzeptabel bis gut $\left(\chi^{2}(166, n=81)=\right.$ 310,$58 ; p<0,001 ; \chi^{2} / \mathrm{df}=0,49 ;$ RMSEA $=0,07 ; \mathrm{CFI}=0,97$; $\mathrm{TLI}=0,96) \mathrm{zu}$ interpretieren. Alle Faktoren der ersten Ordnung luden stark auf dem Faktor der höheren Ordnung (Ladungen zwischen 0,85 und 1,47). Außerdem zeigte sich, dass die höheren Faktorstrukturen signifikante Anteile der Varianz erklärten $\left(\mathrm{R}^{2}\right.$ : Kontamination $=0,66$, Verantwortlichkeit $=0,19$, Inakzeptable Gedanken $=0,95$ und Symmetrie $=0,55$ ). Auch hier führte die höhere Ordnung nicht zu einer signifikanten Abnahme der Modellanpassung. Demnach gibt das Modell die Korrelationen zwischen den Faktoren erster Ordnung gut wieder. Die Ladungen und Standardfehler des finalen Modells sind in Tabelle 2 dargestellt.

\section{Reliabilität}

Alle 20 Items weisen Trennschärfen von $r_{j t}>0,5$ auf. Cronbachs $\alpha$ für die DOCS-Gesamtskala liegt zwischen $\alpha$ $=0,9$ und $\alpha=0,94$. Die Werte der internen Konsistenz für die Subskalen sind Tabelle 3 zu entnehmen. Alle bivariaten Zusammenhänge zwischen den DOCS-Subskalen und dem DOCS-Gesamtscore waren signifikant. Die Test-Retest-Reliabilität des DOCS-Gesamtwertes wurde mithilfe der Daten der nicht-klinischen Stichprobe $(n=$ 
Tabelle 4. Korrelationen DOCS-Gesamtwert und weitere Fragebogeninventare

\begin{tabular}{lllll}
\hline \multicolumn{2}{c}{ Gruppe } & & \\
\cline { 2 - 5 } Messinstrument & Zwangsstörung & Angststörung & Depression & Kontrollgruppe \\
\hline Konstruktnahe Messinstrumente & & & \\
Y-BOCS & $0,57^{*}$ & - & - & - \\
OCI-R & $0,40^{*}$ & $0,77^{*}$ & $0,59^{*}$ & $0,71^{*}$ \\
PI-PR & $0,71^{*}$ & $0,72^{*}$ & $0,76^{*}$ & $0,76^{*}$ \\
\hline Konstruktferne Messinstrumente & & & $0,52^{*}$ \\
BAI & $0,59^{*}$ & 0,39 & $0,52^{*}$ & $0,52^{*}$ \\
BDI & $0,46^{*}$ & 0,20 & $0,48^{*}$ & $0,52^{*}$ \\
SIAS & $0,59^{*}$ & 0,39 & $0,52^{*}$ & \\
\hline
\end{tabular}

Dargestellt sind die Korrelationskoeffizienten $(\rho)$. Der Zusammenhang zur Y-BOCS wird allein für die Stichprobe mit Zwangsstörung angegeben. ${ }^{*} p<0,008$ bzw. $p<0,01$ (Bonferroni-korrigiertes Signifikanzniveau; $p<$ 0,008 für die Gruppe mit Zwangsstörung, $p<0,01$ für die Gruppen mit Angststörung, Depression und Kontrollgruppe).

107) berechnet. Dabei zeigte sich, dass sich der Gesamtwert von 11,38 $(\mathrm{SD}=9,44)$ zu Zeitpunkt 1 und der Gesamtwert von $10,75(S D=8,70)$ nicht signifikant über das zeitliche Fenster von drei bis zwölf Wochen unterschie$\operatorname{den}(t(106)=1,13 ; p=0,26)$. Der Pearson-Korrelationskoeffizient zwischen den beiden Messzeitpunkten kann mit $r=0,8$ als hoch interpretiert werden.

\section{Validität}

Konstruktvalidität

Wie in Tabelle $4 \mathrm{zu}$ sehen ist, liegen die Korrelationskoeffizienten der DOCS mit den konstruktnahen Fragebogeninventaren (Y-BOCS, OCI-R und PI-PR) zwischen $\rho=0,4(p<0,001)$ und $\rho=0,76(p<0,001)$ und mit den konstruktfernen Instrumenten (BAI, BDI-II, SIAS) zwischen $\rho=0,20(p=0,3)$ und $\rho=0,59(p<0,001)$.

$\mathrm{Ob}$ die Korrelationskoeffizienten der konvergenten Validität sich signifikant von denen der diskriminanten Validität unterscheiden, wurde über eine $z$-Prüfgröße bei einseitiger Testung berechnet. Hierfür wurden die Korrelationskoeffizienten zwischen der DOCS und den konstruktnahen und konstruktfernen Fragebogeninventaren $z$-standardisiert und dann Mittelwerte für alle konstruktnahen und Mittelwerte für alle konstruktfernen Korrelationskoeffizienten für jede Gruppe gebildet. Für den Signifikanztest wurde nach Meng et al. [1992] berücksichtigt, dass die zu vergleichenden Korrelationen ebenfalls miteinander korreliert sind, weswegen die $z$-Prüfgröße berechnet wurde. Der Vergleich ergab signifikante Unterschiede für die Subgruppe der PatientInnen mit Angststörungen $(z=2,29 ; p=0,01)$ und für die nicht-klinische Stichprobe $(z=3,71 ; p<0,001)$, aber keine signifikanten Unterschiede für PatientInnen mit Zwangsstörungen $(z=0,26 ; p=0,39)$ und Depression $(z=1,21 ; p=0,11)$. Die Korrelationen der DOCS-Subskalen mit den jeweils konstruktnahen Subskalen zwischen DOCS und OCI-R und des PI-PR fallen für die nicht-klinische Stichprobe und für die Gruppe der ZwangspatientInnen moderat bis hoch aus (Tabelle 5).

Kriteriumsvalidität

Ein Vergleich der Mittelwerte der DOCS-Gesamtwerte mittels einfaktorieller Varianzanalyse ergab einen signifikanten Haupteffekt der Gruppe $(F(3 ; 40,07)=8.91$; $\eta_{\mathrm{p}}{ }^{2}=0,15 ; p<0,001$ ). Post-hoc-Tests (Tukey Honest Significant Difference) zeigen, dass die Gruppe der PatientInnen mit Diagnose Zwangsstörungen höhere Gesamtwerte erzielte $(\mathrm{M}=28,5 ; \mathrm{SD}=17,52)$ als PatientInnen mit Angststörungen $(\mathrm{M}=13,37$; $\mathrm{SD}=11,04 ; p<0,001)$, Depression $(M=14,30$; $S D=11,56 ; p<0,001)$ und die nichtklinische Stichprobe $(M=11,85 ; S D=9,09 ; p<0,001)$. Die Werte der Subgruppen der PatientInnen mit Angststörungen, Depression und der nicht-klinischen TeilnehmerInnen unterscheiden sich nicht signifikant (Bonferroni-korrigiertes Signifikanzniveau von $p=0,008$ : $p s>$ $0,6)$.

Es wurden vier einfaktorielle Varianzanalysen für jede Subskala (Verantwortung, Symmetrie, Inakzeptable Gedanken und Kontamination) mit dem ZwischenSubjektfaktor Gruppenzugehörigkeit (Zwangsstörung, Angststörung, Depression und Kontrollgruppe) berechnet. Die Gruppen hatten signifikant unterschiedliche Werte auf den Subskalen Kontamination $(F(2 ; 123)=$ 9,$\left.49 ; \eta^{2}=0,13 ; p<0,001\right)$, Inakzeptable Gedanken $(F(2$; 123) $\left.=3,09 ; \eta^{2}=0,048 ; p=0,049\right)$ und Verantwortung $\left(F(2 ; 123)=7,104, \eta^{2}=0,104 ; p=0,014\right)$, nicht aber auf der Subskala Symmetrie $\left(F(2 ; 123)=1,2 ; \eta^{2}=0,02 ; p=\right.$ $0,304)$. Geplante Kontraste zeigten für alle vier Subskalen an, dass die Gruppe der PatientInnen mit Zwangsstörung höhere Werte angab als die Gruppe der Patien- 
Tabelle 5. Zusammenhänge der DOCS-Subskalen mit konstruktnahen Skalen des OCI-R und PI-PR

\begin{tabular}{|c|c|c|c|c|}
\hline \multirow[b]{2}{*}{ Skala } & \multicolumn{4}{|l|}{ Gruppe } \\
\hline & Zwang & Angst & Depression & Kontrollgruppe \\
\hline \multicolumn{5}{|l|}{ DOCS und OCI-R } \\
\hline Verantwortung und Kontrollieren & $0,31^{*}$ & 0,40 & 0,42 & $0,49 *$ \\
\hline Symmetrie und Ordnen & $0,78^{*}$ & $0,69^{*}$ & $0,54^{*}$ & $0,73^{*}$ \\
\hline Inakzeptable Gedanken und Zwangsgedanken & 0,26 & $0,76^{*}$ & $0,67 *$ & $0,59 *$ \\
\hline Kontamination und Waschen & $0,52 *$ & 0,44 & $0,68^{*}$ & $0,59^{*}$ \\
\hline \multicolumn{5}{|l|}{ DOCS und PI-PR } \\
\hline Verantwortung und Kontrollieren & $0,70^{*}$ & $0,68^{*}$ & $0,70^{*}$ & $0,58^{*}$ \\
\hline Symmetrie und Zahlen & $0,33 *$ & $0,54^{*}$ & 0,41 & $0,65^{*}$ \\
\hline Symmetrie und Ankleiden & $0,49^{*}$ & 0,59 & 0,37 & $0,54^{*}$ \\
\hline Inakzeptable Gedanken und Impulse & $0,30 *$ & 0,20 & 0,29 & $0,34^{*}$ \\
\hline Inakzeptable Gedanken und Rumination & $0,46^{*}$ & $0,59^{*}$ & 0,39 & $0,52^{*}$ \\
\hline Kontamination und Waschen & $0,87^{*}$ & 0,49 & $0,60^{*}$ & $0,65^{*}$ \\
\hline
\end{tabular}

Dargestellt sind die Korrelationskoeffizienten der vier DOCS-Subskalen mit den konstruktnahen Subskalen des OCI-R und des PI-PR für jede der vier Stichprobengruppen. ${ }^{*} p<0,003$ (Bonferroni-korrigiertes Signifikanzniveau).

tInnen mit Angststörung, Depression und die nicht-klinische Stichprobe ( $p s<0,007)$. Die Gruppen der PatientInnen mit Depression und Angststörung und die nicht-klinische Stichprobe unterscheiden sich nicht signifikant.

\section{Diagnostische Genauigkeit}

Um zu prüfen, inwieweit sich die diagnostische Genauigkeit für die Gruppe der PatientInnen mit Zwangsstörung von (a) der Gruppe der Angst- oder depressiven PatientInnen und (b) der nicht-klinische Kontrollgruppe unterschied, wurden ROC-Analysen getrennt für den Gesamtwert und die Subskalen der DOCS berechnet. Bei der Prüfung der Diskriminierungsfähigkeit der DOCS zwischen PatientInnen mit Zwangsstörung und PatientInnen mit Angststörungen oder Depressionen zeigten sich AUC-Schätzungen von 0,59 (Subskala Symmetrie) bis 0,7 (Subskala Kontamination). Für den DOCS-Gesamtwert ergab sich im Vergleich zu den Subskalen die höchste AUC-Schätzung (AUC $=0,75$; 95\% Konfidenzintervall $=0,68-0,82)$. Bei der Unterscheidung der Gruppe der PatientInnen mit Zwangsstörung von den nicht-klinischen TeilnehmerInnen zeigten sich AUC-Schätzungen von 0,54 (Subskala Kontamination) bis 0,67 (Subskala Inakzeptable Gedanken). Für den DOCS-Gesamtwert ergab sich im Vergleich zu den Subskalen die höchste AUC-Schätzung $($ AUC $=0,80 ; 95 \%$ Konfidenzintervall $=$ 0,75-0,85). Die Ergebnisse zeigen, dass der Gesamtwert der DOCS-PatientInnen mit Zwangsstörungen zufriedenstellend von PatientInnen mit Angststörungen oder Depressionen sowie von der nicht-klinischen Stichprobe unterschieden werden kann.

Deutschsprachige Validierung der DOCS
Der Vergleich der Diskriminierungsfähigkeit zwischen DOCS und OCI-R ergab signifikant größere AUCSchätzer für den DOCS-Gesamtwert (Subgruppen mit Angststörungen oder Depressionen, DeLong-Test für korrelierte ROC-Kurven: AUC $=0,27, Z=7,002$; $p<$ 0,001, Gruppe mit Zwangsstörung und der nicht-klinischen Kontrollgruppe: $\mathrm{AUC}=0,28, Z=8,002$; $p<$ $0,001)$. Ein direkter Vergleich zwischen DOCS und PI-PR ergab dagegen keinen signifikanten Unterschied der AUC-Schätzer. Demnach scheint die DOCS für die oben genannten Vergleiche eine größere diagnostische Genauigkeit aufzuweisen als der OCI-R, nicht aber als der PIPR-Gesamtwert (beides Abb. 1).

Entsprechend den Berechnungen liegt der optimale Cut-Off-Wert für die Unterscheidung von PatientInnen mit Zwangsstörung von PatientInnen mit Angststörung oder Depression bei 18. 65\% der PatientInnen mit Zwangsstörung (Sensitivität) und 66\% der PatientInnen mit Angststörung oder Depression (Spezifität) werden anhand dieses Wertes korrekt identifiziert. Für die Unterscheidung von PatientInnen mit Zwangsstörung von der nicht-klinischen Kontrollgruppe liegt der optimale Cut-Off-Wert bei 16 . Hierbei werden $72 \%$ der PatientInnen mit Zwangsstörung (Sensitivität) und 71\% der Kontrollgruppe (Spezifität) korrekt identifiziert.

\section{Diskussion}

In der vorliegenden Studie wurden die psychometrischen Eigenschaften der deutschsprachigen Version der DOCS in Stichproben von PatientInnen mit 


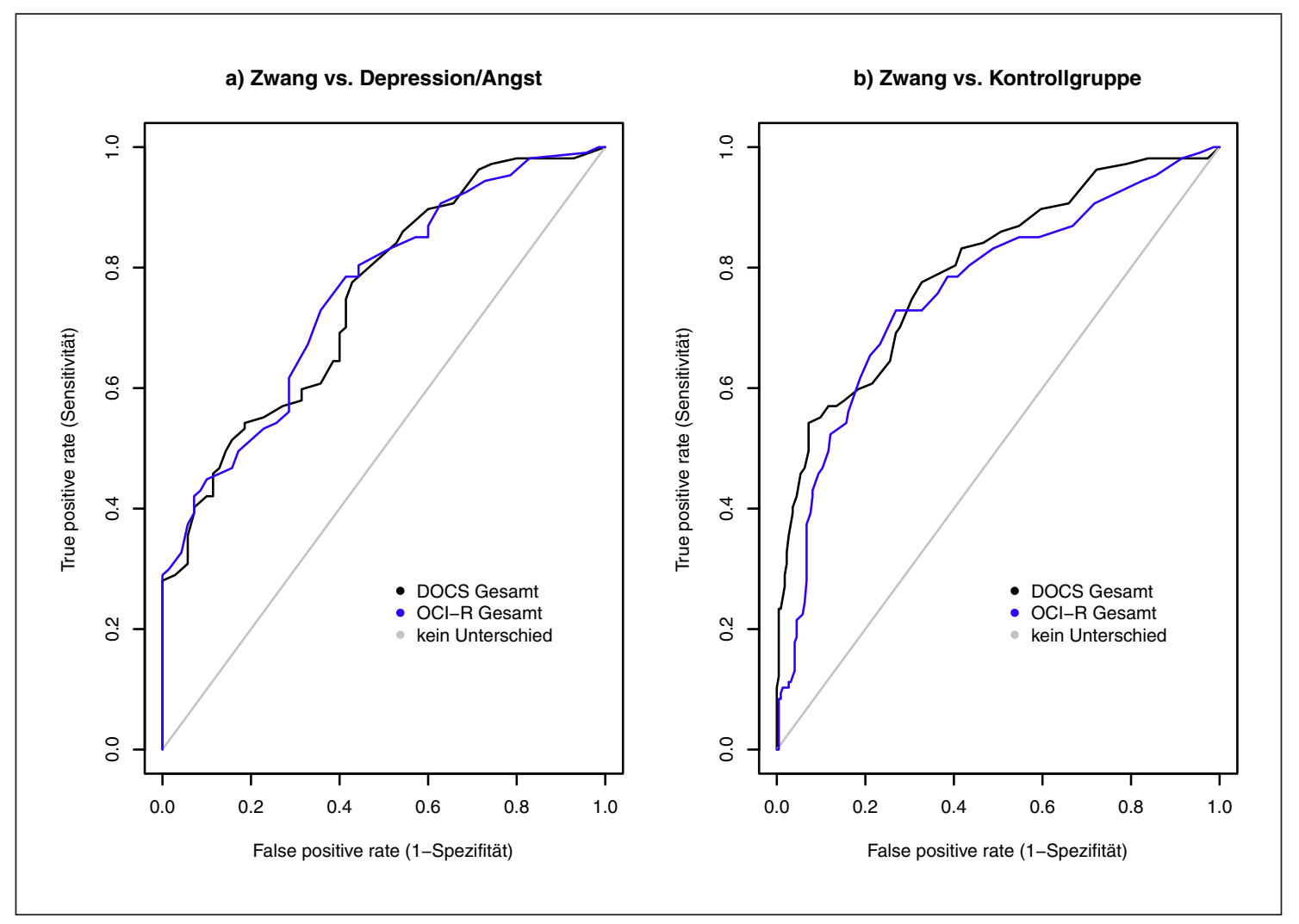

Abb. 1. Receiver Operating Characteristic-Kurven (ROC-Kurven) für den Vergleich zwischen den PatientInnen mit Zwangsstörungen und den PatientInnen mit Depression und Angst auf der linken Seite (a) und Kurven für den Vergleich zwischen PatientInnen mit Zwangsstörungen und der nicht-klinischen Kontrollgruppe auf der rechten Seite (b).

Zwangsstörung, Angststörung und Depression sowie nicht-klinischen TeilnehmerInnen überprüft. Insgesamt weist die deutschsprachige Version der DOCS akzeptable bis gute psychometrische Eigenschaften auf und ist damit vergleichbar mit der Originalversion und den bisherigen Übersetzungen [Abramowitz et al., 2010; López-Solà et al., 2014]. Die DOCS ist mit ihren 20 Items und durch ihren Verzicht auf eine umfangreiche Symptomliste ein Instrument, das eine relativ ökonomische Erfassung von Zwangssymptomen ermöglicht. Dennoch fielen bestimmte Kennwerte im Rahmen der EFA und der Konstruktvalidität etwas geringer als in den Vergleichsstudien aus. Entsprechend unserer Erwartungen zeigt die robuste und reliable deutschsprachige DOCS eine große Konstruktnähe zum PI-PR und eine höhere diagnostische Genauigkeit als der OCI-R-Fragebogen.

Unsere Ergebnisse zeigen, dass auch die deutsche Langversion der DOCS in klinischen und nicht-klinischen Stichproben eine Vier-Faktoren-Struktur aufweist. Die Ergebnisse sind robust und zeigten eine akzeptable bis gute Modellgüte. Die Abweichungen hinsichtlich der EFA könnten auf die deutlich kleinere nicht-klinische Kontrollstichprobe im Vergleich zur Ori- ginalpublikation von Abramowitz et al. [2010] $(n=223$ vs. $n=1$ '044) zurückzuführen sein. Die Faktorenstruktur kann trotzdem als robust interpretiert werden, da sowohl die KFA für den zweiten Teil der nicht-klinischen Stichprobe wie auch die KFA für die zusammengefassten klinischen Stichproben eine vergleichbare Modellgüte zur Originalarbeit aufweisen.

Die Mittelwerte und Standardabweichungen für den Gesamtwert und die Subskalen waren denjenigen sehr ähnlich, die für die Originalversion berichtet wurden [Abramowitz et al., 2010]. Dieses Ergebnis zeigt im Zusammenhang mit den sehr guten Kennwerten der Reliabilität (Cronbachs $\alpha$ und die Test-Retest-Reliabilität) der deutschsprachigen DOCS, dass sowohl ein stabiler Gesamtwert als auch stabile Werte für die Subskalen berechnet werden können. In Anbetracht ähnlicher Ergebnisse der bisherigen Übersetzungen [Wang et al., 2012; Ólafsson et al., 2013; López-Solà et al., 2014; Melli et al., 2015] wird somit auch die länderübergreifende Verwendbarkeit der DOCS für die deutschsprachige Version bestätigt.

Die internen Konsistenzschätzungen für die Gesamtund Subskalenwerte reichten von befriedigend bis gut. Wie erwartet waren für die nicht-klinische Kontroll- 
stichprobe und die Gruppe der PatientInnen mit Angststörungen die Korrelationen zwischen dem DOCS-Gesamtwert und den konstruktfernen Messinstrumenten (BAI, BDI, SIAS) geringer als mit den konstruktnahen Messinstrumenten (PI-PR und OCI-R). Dieser Unterschied zeigte sich allerdings für die Gruppen der PatientInnen mit Zwangsstörungen und Depressionen nicht. Dies könnte auch daran liegen, dass viele Personen mit komorbiden Depressionen in der Gruppe der PatientInnen mit Zwangsstörungen waren, was sich auch in hohen BDI Werten zeigte. Auf eine weitere Ursache könnte der geringe bivariate Zusammenhang zwischen der DOCS und dem konstruktnahen OCI-R hinweisen. Da der OCI-R auch eine Skala zum Horten beinhaltet, könnte diese Subskala für die niedrige Korrelation zumindest mitverantwortlich sein. Dennoch ergibt sich insgesamt eine positive Beurteilung der Konstruktvalidität, da, wie erwartet, signifikante und hohe Zusammenhänge zwischen dem DOCS-Gesamtwert und den Gesamtwerten des PI-PR und der Y-BOCS in der Gruppe der PatientInnen mit Zwangsstörungen vorlagen. Bemerkenswert, auch im Vergleich zur Studie von Abramowitz et al. [2010], sind aber vor allem die unterschiedlichen Befunde zwischen den PatientInnen mit Angststörungen und den PatientInnen mit Depressionen. Dieser Unterschied zeigt, wie wichtig beide Störungsgruppen zum Vergleich bei einer solchen Validierungsstudie sind.

Bei den Ergebnissen zur Konstruktvalidität auf Skalenebene finden sich bei der Gruppe der PatientInnen mit Zwangsstörungen hohe Zusammenhänge zwischen den DOCS-Subskalen Kontamination und Symmetrie und dem OCI-R bzw. dem PI-PR andererseits. Dagegen scheint vor allem die Subskala Inakzeptable Gedanken wenig mit den konstruktnahen Subskalen des OCI-R und des PI-PR zusammenzuhängen. Dabei weist die DOCSSkala Inakzeptable Gedanken eine etwas bessere Konstruktvalidität mit der PI-PR-Ruminationsskala ("Wenn ich beginne, an bestimmte Dinge zu denken, werde ich von den Gedanken völlig eingenommen und kann an nichts anderes mehr denken") auf als mit der PI-PR-Impulsskala ("Wenn ich von einer Brücke oder aus einem hohen Fenster hinabsehe, verspüre ich einen Impuls, mich in die Tiefe zu stürzen"). Möglicherweise fällt die Selbsteinschätzung der Zeit und des Ausmaßes von Impulsen im Rahmen der DOCS schwerer als die Einschätzung über eine relativ unspezifische Likert-Skala im Rahmen des OCI-R und des PI-PR.

Die Kriteriumsvalidität fiel dagegen, wie oben bereits beschrieben, vergleichbar gut wie in der Originalarbeit aus. Insbesondere zeigten sich durchgehend höhere Werte auf dem Gesamtscore wie auch auf allen Subskalen bei der Gruppe mit Zwangsstörungen im Vergleich zu allen anderen Gruppen.

Deutschsprachige Validierung der DOCS
Für die Unterscheidung der klinischen Gruppen (Zwangsstörung vs. Depression/Angststörung) fiel die diagnostische Genauigkeit etwas geringer aus, als die Ergebnisse der Originalarbeit dies nahelegen. Die diagnostische Genauigkeit zur Unterscheidung von PatientInnen mit Zwangsstörungen und der nicht-klinischen Kontrollstichprobe fiel dagegen vergleichbar gut aus. Insgesamt fiel die Sensitivität und die Spezifität insbesondere für die Unterscheidung der PatientInnengruppen (Zwangsstörung vs. Depression/Angststörung) geringer aus als in der Originalarbeit. Dies zeigt sich in einem niedrigeren CutOff-Wert in der vorliegenden Studie (18 vs. 21 in der Originalstichprobe, nur Angststörung). Zur Unterscheidung der ZwangspatientInnen mit der nicht-klinischen Kontrollstichprobe waren Sensitivität und Spezifität sowie der daraus resultierende Cut-Off-Wert vergleichbar mit den Werten der Originalstichprobe (16 vs. 18 in der Originalstichprobe). Insgesamt zeigt sich ähnlich wie in anderen Validierungsstudien zur DOCS [Abramowitz et al., 2010; López-Solà et al., 2014] eine höhere Sensitivität der DOCS im Vergleich zum OCI-R, nicht aber im Vergleich zum PI-PR. Hierbei waren die Werte zur diagnostischen Genauigkeit des OCI-R in unserer Untersuchung deutlich schlechter als in der Arbeit von Abramowitz et al. [2010]. Dies könnte, zusammengenommen mit den oben beschriebenen Ergebnissen, auch auf eine generelle Verzerrung in den Antworten des OCI-R in der vorliegenden Studie hinweisen.

Auch wenn die DOCS durch ihre Verbindung aus dimensionalem und kategorialem Ansatz die neuere Forschung zu Zwangsstörungen besser abbildet und dadurch auch bei Menschen mit geringeren Einschränkungen differenzieren kann, ist das Verfahren nicht ohne Limitationen. So kann es sein, dass Menschen, die in einer Domäne schwer beeinträchtigt sind, geringere Gesamtwerte in der DOCS erreichen als Menschen, die in mehreren Domänen leichtere Beeinträchtigungen aufweisen. Hier empfehlen wir daher, immer auch die Werte der Subkategorien bei der Schweregradbeurteilung mit zu betrachten. $\mathrm{Zu}$ den Limitationen der vorliegenden Studie gehört zweitens, dass die Gruppen der PatientInnen mit Angststörungen und Depression deutlich kleiner waren als die der PatientInnen mit der Diagnose Zwangsstörung. Drittens konnte die Änderungssensitivität aufgrund zu geringer Fallzahlen noch nicht beurteilt werden. Viertens könnte eine Limitation der Studie sein, dass die Daten der nicht-klinischen TeilnehmerInnen im Internet erhoben wurden, während die klinischen Stichproben die Fragebögen per Paper-Pencil-Verfahren durchführten. Allerdings zeigte sich in vergleichenden Untersuchungen, dass Fragebögen unabhängig von der verwendeten Erhebungsmethode ähnliche Mittelwerte sowie ähnliche interne Konsistenzen und Intra-Korrelations-Koeffizienten auf- 
zeigten [Coles et al., 2007]. Eine fünfte Limitation der Studie ist, dass sich Alter und Geschlechterverteilung zwischen den Gruppen unterschieden. Dies war zwar in der Originalstudie von Abramowitz et al. [2010] auch der Fall, trotzdem könnte es sein, dass die Gruppenunterschiede dieser beiden Variablen die Ergebnisse verzerrt haben. So könnte es sein, dass beispielsweise die diagnostische Genauigkeit zwischen den klinischen Gruppen auch durch unterschiedliche Alterseffekte verzerrt wurde.

Zusammenfassend ist die deutschsprachige DOCS aber ein robustes, reliables und erstmals validiertes Messinstrument zur dimensionalen Erfassung von Zwangssymptomen, das bisherigen Limitationen der Fragebogen- und Selbstbeurteilungsdiagnostik von Zwangsstörungen durch die Verknüpfung von dimensionaler und kategorialer Selbstbeurteilungsdiagnostik entgegenwirken kann.

\section{Danksagung}

Wir bedanken uns bei allen Teilnehmern und Teilnehmerinnen an der Studie. Wir bedanken uns bei Amal Kebir, Neeske Beckmann, Sina Klüver, Hille Stühring, Eva Mosig, den Therapeuten und Therapeutinnen an der Psychotherapeutischen Hochschulambulanz der Universität Leipzig und am Leipziger Ausbildungsinstitut für Psychologische Psychotherapie sowie den MitarbeiternundMitarbeiterinnen derPsychosomatischenSchön-Klinik Bad Bramstedt und des Helios Parkkrankenhaus Leipzig für die Unterstützung bei der Datenerhebung. Außerdem danken wir Jonathan Abramowitz für den zügigen Vergleich unserer Rückübersetzung mit der Originalversion.

\section{Ethische Aspekte}

Alle TeilnehmerInnen wurden über die Ziele und Abläufe der Studie aufgeklärt und gaben ihr Einverständnis zur Teilnahme an der Studie. Es liegt ein positives Votum der Ethikkommission der Medizinischen Fakultät der Universität Leipzig vom 23.06.2016 vor (217/16-ek).

\section{Interessenkonflikt}

Die Autoren erklären, dass kein Interessenkonflikt besteht.

\section{Finanzielle Unterstützung}

Diese Arbeit erhielt keine spezifischen Zuschüsse von Förderorganisationen aus dem öffentlichen, kommerziellen oder gemeinnützigen Sektor. Die Autoren werden durch die Deutsche Forschungsgemeinschaft (DFG) und die Universität Leipzig im Rahmen des Programms Open Access Publishing unterstützt.

\section{Autorenmitwirkung}

Die Studie wurde von C.E. entwickelt und im Rahmen ihres Lehrstuhls gefördert. Das Manuskript wurde von ihr überarbeitet. J.F.-L. und I.J. haben in dieser Studie federführend die Rekrutierung durchgeführt, die Daten ausgewertet und den ersten Entwurf des Manuskripts verfasst. I.J. ist psychologische Leiterin der Zwangsambulanz, in der ein Großteil der Zwangspatienten rekrutiert wurden. K.S. ist Leiterin dieser Klinik und hat die Rekrutierung gefördert. C.S. hat große Teile der weiteren klinischen Stichprobe rekrutiert. T.L. hat den Fragebogen aus dem Englischen ins Deutsche übersetzt und an der Überarbeitung des Manuskripts mitgewirkt. F.K. hat das Manuskript substantiell überarbeitet.

\section{Literatur}

Abramowitz JS, Deacon BJ, Olatunji BO, Wheaton MG, Berman NC, Losardo D, et al. Assessment of obsessive-compulsive symptom dimensions: development and evaluation of the Dimensional Obsessive-Compulsive Scale. Psychol Assess. 2010 Mar;22(1):180-98.

American Psychiatric Association. Diagnostic and Statistical Manual of Mental Disorders: Diagnostic and Statistical Manual of Mental Disorders. Arlington (VA): American Psychiatric Association; 2013.

Beck AT, Epstein N, Brown G, Steer RA. An inventory for measuring clinical anxiety: psychometric properties. J Consult Clin Psychol. 1988 Dec;56(6):893-7.

Beck AT, Ward CH, Mendelson M, Mock J, Erbaugh J. An inventory for measuring depression. Arch Gen Psychiatry. 1961 Jun;4(6): 561-71.

Bollen KA, editor. Structural Equations with Latent Variables. Hoboken, NJ, USA: John Wiley \& Sons, Inc; 1989. https://doi. org/10.1002/9781118619179.

Brislin RW. Back-translation for cross-cultural research. J Cross Cult Psychol. 1970;1(3): 185-216.
Brown TA. Confirmatory factor analysis for applied research. New York, NY, Guilford; 2006

Cohen J. Statistical power analysis for the behavioral sciences. Hillsdale (N.J.): L. Erlbaum Associates; 1988.

Coles ME, Cook LM, Blake TR. Assessing obsessive compulsive symptoms and cognitions on the internet: evidence for the comparability of paper and Internet administration. Behav Res Ther. 2007 Sep;45(9):2232-40.

Cronbach LJ. Coefficient alpha and the internal structure of tests. Psychometrika. 1951;16(3): 297-334.

Field AP. Discovering statistics with SPSS. London: Sage; 2014.

Foa EB, Huppert JD, Leiberg S, Langner R, Kichic R, Hajcak G, et al. The Obsessive-Compulsive Inventory: development and validation of a short version. Psychol Assess. 2002 Dec;14(4): 485-96.

Gönner S, Ecker W, Leonhart R. The Padua Inventory: do revisions need revision? Assessment. 2010 Mar;17(1):89-106.

Gönner S, Leonhart R, Ecker W. Das Zwangsinventar OCI-R - die deutsche Version des Obsessive-Compulsive Inventory-Revised - Ein kurzes Selbstbeurteilungsinstrument zur mehrdimensionalen Messung von Zwangssymptomen. Psychother Psychosom Med Psychol. 2007 Sep-Oct;57(9-10):395-404.

Goodman WK, Price LH, Rasmussen SA, Mazure C, Delgado P, Heninger GR, et al. The YaleBrown Obsessive Compulsive Scale. II. Validity. Arch Gen Psychiatry. 1989a Nov;46(11): 1012-6.

Goodman WK, Price LH, Rasmussen SA, Mazure C, Fleischmann RL, Hill CL, et al. The YaleBrown Obsessive Compulsive Scale. I. Development, use, and reliability. Arch Gen Psychiatry. 1989b Nov;46(11):1006-11.

Guadagnoli E, Velicer WF. Relation of sample size to the stability of component patterns. Psychol Bull. 1988 Mar;103(2):265-75.

Hand I, Büttner-Westphal H. Die Yale-Brown Obsessive Compulsive Scale (Y-BOCS): Ein halbstrukturiertes Interview zur Beurteilung des Schweregrades von Denk- und Handlungszwängen. Verhaltenstherapie. 1991; 1(3):223-5.

Hautzinger M, Keller F, Kühner C. Beck Depressionsinventar, 2. Revision (BDI-II). Frankfurt. Harcourt Test Services; 2006. 
Heyman I, Fombonne E, Simmons H, Ford T, Meltzer H, Goodman R. Prevalence of obsessive-compulsive disorder in the British nationwide survey of child mental health. $\mathrm{Br} J$ Psychiatry. 2001 Oct;179(4):324-9.

Hooper D, Coughlan J, Mullen MR. Structural Equation Modelling: Guidelines for Determin-ing Model Fit. Electron J Bus Res Methods. 2008;6:53-60.

Hu LT, Bentler PM. Cutoff criteria for fit indexes in covariance structure analysis: conventional criteria versus new alternatives. Struct Equ Modeling. 1999;6(1):1-55.

Jacobsen D, Kloss M, Fricke S, Hand I, Moritz S. Reliabilität der deutschen Version der YaleBrown Obsessive Compulsive Scale. Verhaltenstherapie. 2003;13(2):111-3.

Kessler RC, Chiu WT, Demler O, Merikangas KR, Walters EE. Prevalence, severity, and comorbidity of 12-month DSM-IV disorders in the National Comorbidity Survey Replication. Arch Gen Psychiatry. 2005 Jun;62(6):617-27.

Klepsch R, Zaworka W, Hand I, Lünenschloss K, Jauernig G. Derivation and validation of the Hamburg Obsession/Compulsion Inventory - Short Form (HOCI-S): first results. Psychol Assess. 1991;3(2):196-201.

Kühner C, Bürger C, Keller F, Hautzinger M. Reliabilität und Validität des revidierten BeckDepressionsinventars (BDI-II). Befunde aus deutschsprachigen Stichproben. Nervenarzt. 2007 Jun;78(6):651-6.

López-Solà C, Gutiérrez F, Alonso P, Rosado S, Taberner J, Segalàs C, et al. Spanish version of the Dimensional Obsessive-Compulsive Scale (DOCS): psychometric properties and relation to obsessive beliefs. Compr Psychiatry. 2014 Jan;55(1):206-14

Mataix-Cols D, Rosario-Campos MC, Leckman JF. A multidimensional model of obsessivecompulsive disorder. Am J Psychiatry. 2005 Feb;162(2):228-38
Mattick RP, Clarke JC. Development and validation of measures of social phobia scrutiny fear and social interaction anxiety. Behav Res Ther. 1998 Apr;36(4):455-70.

McKay D, Abramowitz JS, Calamari JE, Kyrios M, Radomsky A, Sookman D, et al. A critical evaluation of obsessive-compulsive disorder subtypes: symptoms versus mechanisms. Clin Psychol Rev. 2004 Jul;24(3):283-313.

Melli G, Chiorri C, Bulli F, Carraresi C, Stopani E, Abramowitz JS. Factor Congruence and Psychometric Properties of the Italian Version of the Dimensional Obsessive-Compulsive Scale (DOCS) Across Non-Clinical and Clinical Samples. J Psychopathol Behav Assess. 2015; 37(2):329-39.

Meng X, Rosenthal R, Rubin DB. Comparing correlated correlation coefficients. Psychol Bull. 1992;111(1):172-5.

Ólafsson RP, Arngrímsson JB, Árnason P, Kolbeinsson P, Emmelkamp PM, Kristjánsson Á, et al. The Icelandic version of the dimensional obsessive compulsive scale (DOCS) and its relationship with obsessive beliefs. J Obsessive Compuls Relat Disord. 2013;2(2):14956.

Prinz M, Petermann F. Beck Angst-Inventar (BAI). Z Klin Psychol Psychopathol. 2009;57: 63-6.

R Core Team. R: A language and environment for statistical computing. Vienna, Austria: R Foundation for Statistical Computing; 2019.

Revelle W. psych: Procedures for Personality and Psychological Research. Evanston, Illinois, USA, Northwestern University, 2019.

Rosseel Y. lavaan: An R Package for Structural Equation Modeling. J Stat Softw. 2012;48(2): $1-36$.

Ruscio AM, Stein DJ, Chiu WT, Kessler RC. The epidemiology of obsessive-compulsive disorder in the National Comorbidity Survey Replication. Mol Psychiatry. 2010 Jan;15(1):5363.
Sanavio E. Obsessions and compulsions: the Padua Inventory. Behav Res Ther. 1988;26(2): 169-77.

Schaible R, Armbrust M, Nutzinger DO. YaleBrown Obsessive Compulsive Scale: Sind Selbst- und Fremdrating äquivalent? Verhaltenstherapie. 2001;11(4):298-303.

Stangier U, Heidenreich T, Berardi A, Golbs U, Hoyer J. Die Erfassung sozialer Phobie durch die Social Interaction Anxiety Scale (SIAS) und die Social Phobia Scale (SPS). Z Klin Psychol Psychopathol Psychother. 1999;28(1): 28-36.

Storch EA, Rasmussen SA, Price LH, Larson MJ, Murphy TK, Goodman WK. Development and psychometric evaluation of the YaleBrown Obsessive-Compulsive Scale - Second Edition. Psychol Assess. 2010 Jun;22(2):22332.

Wang CY, Wang JP, Tang T, Zhang SY, Bjorgvinsson T. Psychometric validation of dimensional obsessive-compulsive scale (DOCS) in Chinese college students. Chin J Clin Psychol. 2012;20:315-9.

Wirtz M. Über das Problem fehlender Werte: wie der Einfluss fehlender Informationen auf Analyseergebnisse entdeckt und reduziert werden kann. Rehabilitation (Stuttg). 2004 Apr;43(2):109-15.

Wittchen HU, Wunderlich U, Gruschwitz S, Zaudig M. SKID I. Strukturiertes Klinisches Interview für DSM-IV. Achse I: Psychische Störungen. Interviewheft und Beurteilungsheft. Eine deutschsprachige, erweiterte Bearb. d. amerikanischen Originalversion des SKID I. Göttingen: Hogrefe; 1997.

Woody SR, Steketee G, Chambless DL. Reliability and validity of the Yale-Brown ObsessiveCompulsive Scale. Behav Res Ther. 1995 Jun; 33(5):597-605.

Zaworka W, Hand I, Lünenschloss K, Jauernig G. Das Hamburger Zwangsinventar. Weinheim. Beltz; 1983. 